\title{
Beta blocker use is associated with an improvement in intermediate overall survival after Transcatheter Aortic Valve Replacement (TAVR)
}

\author{
Brent Klinkhammer* MD, PharmD
}

\begin{abstract}
Klinkhammer B. Beta blocker use is associated with an improvement in intermediate overall survival after Transcatheter Aortic Valve Replacement (TAVR). Curr Res Cardiol 2018;5(3):34-7.

BACKGROUND: Despite initially concerns about their use, beta-adrenergic blockers (BB) have been associated with improved outcomes in patients with uncorrected aortic stenosis. However, the effect of beta blockers on outcomes after transcatheter aortic valve replacement (TAVR) is unknown.
\end{abstract}

PURPOSE: To elucidate the effect of beta blockers on patient-specific outcomes after TAVR. underwent a TAVR for severe aortic stenosis was performed to determine the effect of beta blockers on outcomes after TAVR.
METHODS: A retrospective cohort study of 339 consecutive patients who

RESULTS: Despite a greater prevalence of several significant comorbidities and a higher predicted surgical risk, beta-adrenergic blockers are associated with an improve in overall survival at 1 year $(83 \%$ vs. $67 \%, p=0.0398)$. The use of beta blocker was also associated with a reduction of major adverse cardiovascular and cerebrovascular events in the short-term (30 days- 6 months) time period ( $23 \%$ vs. $39 \%, \mathrm{p}=0.0208$ )

CONCLUSION: This study gives evidence to suggest that beta blockers are associated with an improvement of overall survival in the intermediate time period. This finding suggests that TAVR may be a compelling indication for use of a beta blocker.

Key Words: Aortic stenosis; Transcatheter aortic valve replacement (TAVR); Myocardial infarction; Hypertension

\section{INTRODUCTION}

$\mathrm{T}^{\mathrm{s}}$ raditionally, beta-adrenergic blockers and other negative inotropic agents have been relatively contraindication in patients with severe aortic stenosis given concerns about reducing left ventricular contractility in the setting of fixed outlet obstruction. However, more recent studies have given evidence to suggest that beta blocker may not be contraindicated and possible even beneficial in patients with uncorrected aortic stenosis (1). In a post hoc analysis published in JAHA in 2017, patients with asymptomatic aortic stenosis on beta blockers was strongly associated with a with lower risk of all-cause mortality and cardiovascular death. Furthermore, in an Italian study published by Rossi et al. beta blocker use was similarity associated with an all-cause reduction in mortality in patients who were initially evaluated as candidates for possible aortic valve replacement but were ultimately not felt to be candidates (2). Taken together these studies suggest that the antiischemic and anti-cardiac remodeling properties of these agents have a role in the treatment of aortic stenosis.

Over much of the last decade, transcatheter aortic valve replacement (TAVR) has emerged as a viable option for nearly all patients with severe aortic stenosis in an attempt to enhance survival and quality of life. This advancement and widespread implementation of TAVR in previously medically unfit patients for surgical aortic valve patients raises questions about the potential role of beta blocker in patients following TAVR. It may be hypothesizing that the benefit of beta blocker in the post-TAVR arena may be even more beneficial than in patients with uncorrected aortic stenosis given the relief of obstruction from TAVR mitigates the major risk of using negative inotropes in these patients. To date, minimal research has been published to answer this important question and there also has been little data from surgical aortic valve replacement to guide potential research. However, in a recent published abstract by Alrifai et al. pre-operative beta blocker use did not improve the overall mortality (3). Similarity, Lindman et al. found no survival benefit at 1 year in patients after TAVR (4). In this trial, we aim to further characterize the impact of beta-adrenergic blockade on clinical outcomes following TAVR. We believe that beta blocker use could lead to similar improvements in overall survival to what has been documented in uncorrected aortic stenosis.

\section{PATIENTS AND METHODS}

A single institution case-control study was conducted using data from large tertiary care referral center. We performed a retrospective chart review of 339 consecutive patients who underwent a transcatheter aortic valve replacement (TAVR) at Sanford Health in Fargo, ND from 8/10/2012 to 11/15/2016 for severe aortic stenosis, defined as an aortic valve area less than $1 \mathrm{~cm}^{2}$. The last date of data acquisition was $1 / 4 / 2017$. The study population was divided in two cohorts based on whether or not they were taking a betaadrenergic antagonist at the time of TAVR. Patients not on a beta-blocker were assigned to the control cohort. Primary outcomes were overall survival at 1 month, 6 months, and 1-year post-TAVR. Secondary outcomes were procedural complications, major adverse cardiovascular and cerebrovascular events (MACCE) defined as death from any cause, myocardial infarction, rehospitalization, or stroke, cardiovascular mortality, myocardial infarction, stroke/TIA, heart failure exacerbation, or rehospitalization for any reason in defined time periods. Pre and postprocedural echocardiographic data were also compared. The clinical outcomes were assessed in accordance with the Consortium-2 (5). Heart failure exacerbation was defined as a gradual or rapid change in heart failure signs and symptoms resulting in a need for a change in therapy or hospitalization.

Informed consent was not required for inclusion in our retrospective study due to the nature of the study, and the absence of any direct interventions. This study protocol received dual IRB approval from the University of North Dakota IRB and from the Sanford Health IRB. The Fisher's exact test was performed to determine statistical significance of categorical data and ANOVA was used to determine the statistical significance of continuous variables. All p-values were two-sided, and p-values $<0.05$ were considered significant.

\section{RESULTS}

A total of 339 patients were included in the analysis. The baseline characterizes of both cohorts are given in Table 1 . standardized endpoint definitions for TAVR of the Valve Academic Research

University of Nebraska Medical Center, Omaha, USA

Correspondence: Dr Brent Klinkhammer MD, PharmD, University of Nebraska Medical Center, 982055 Nebraska Medical Center, Omaha, NE 68198-2055, USA, Telephone 218-261-0967, e-mail brent.klinkhammer@unmc.edu

Received: October 03, 2018, Accepted: October 11, 2018, Published: October 15, 2018

open Access

This open-access article is distributed under the terms of the Creative Commons Attribution Non-Commercial License (CC BY-NC) (http:// creativecommons.org/licenses/by-nc/4.0/), which permits reuse, distribution and reproduction of the article, provided that the original work is properly cited and the reuse is restricted to noncommercial purposes. For commercial reuse, contact reprints@pulsus.com 
TABLE 1

Baseline characteristics

\begin{tabular}{|c|c|c|c|}
\hline Variables & $\begin{array}{l}\text { Beta-Blocker } \\
(n=250)\end{array}$ & $\begin{array}{l}\text { Control } \\
(n=89)\end{array}$ & P-value \\
\hline Age & $79.2(8.6)$ & $79.0(10.0)$ & 0.8867 \\
\hline Male sex & $57(143)$ & $51(45)$ & 0.3207 \\
\hline BMI & $30.7(6.1)$ & $29.7(6.7)$ & 0.1919 \\
\hline Caucasian race & $99(247)$ & $100(89)$ & 0.5696 \\
\hline EuroSCORE (\%) & $9.0(6.3)$ & $7.0(5.7)$ & 0.0105 \\
\hline Preprocedural HTN & $91(227)$ & $80(71)$ & 0.0126 \\
\hline Preprocedural CAD & $78(194)$ & $61(54)$ & 0.0032 \\
\hline $\begin{array}{l}\text { Baseline Ejection Fraction } \\
<40 \%\end{array}$ & $16(40)$ & $10(9)$ & 0.2196 \\
\hline $\begin{array}{c}\text { Preprocedural NYHA } \\
\text { Class III OR IV Symptoms }\end{array}$ & $44(111)$ & $43(38)$ & 0.8047 \\
\hline Preprocedural DM & $38(96)$ & $27(24)$ & 0.0028 \\
\hline Prior Stroke/TIA & $12(30)$ & $10(9)$ & 0.7029 \\
\hline Preprocedural Atrial Fibrillation & $34(84)$ & $21(19)$ & 0.0323 \\
\hline $\begin{array}{l}\text { Preprocedural Serum } \\
\text { Creatinine }(\mathrm{mg} / \mathrm{dL})\end{array}$ & $1.22(0.64)$ & $1.35(1.26)$ & 0.2243 \\
\hline $\begin{array}{c}\text { Preprocedural eGFR }<60 \\
\mathrm{~mL} / \mathrm{min}\end{array}$ & $50(126)$ & $42(37)$ & 0.1746 \\
\hline Preprocedural Dyslipidemia & $92(231)$ & $79(70)$ & 0.0013 \\
\hline Preprocedural AAA & $13(32)$ & $6(5)$ & 0.0745 \\
\hline $\begin{array}{l}\text { Preprocedural Carotid Artery } \\
\text { Stenosis }>50 \% \text { or Prior CEA }\end{array}$ & $28(71)$ & $25(22)$ & 0.5806 \\
\hline $\begin{array}{c}\text { Preprocedural Symptomatic } \\
\text { PAD }\end{array}$ & $27(68)$ & $28(25)$ & 0.8904 \\
\hline Prior CABG & $33(83)$ & $12(11)$ & 0.0001 \\
\hline Prior $\mathrm{PCl}$ & $39(97)$ & $30(27)$ & 0.1614 \\
\hline Prior Permanent Pacemaker & $14(34)$ & $8(7)$ & 0.1868 \\
\hline Prior Aortic Valvuloplasty & $17(43)$ & $18(16)$ & 0.8715 \\
\hline \multicolumn{4}{|c|}{ Cardiovascular Pharmacology } \\
\hline ACE inhibitor & $33(83)$ & $28(25)$ & 0.4275 \\
\hline Angiotensin II receptor blocker & $16(41)$ & $21(19)$ & 0.3321 \\
\hline Calcium channel blocker & $30(76)$ & $24(21)$ & 0.2746 \\
\hline Thiazide diuretic & $17(42)$ & $30(27)$ & 0.0089 \\
\hline Loop diuretic & $54(135)$ & $36(32)$ & 0.0044 \\
\hline Spironolactone & $4(11)$ & $1(1)$ & 0.1957 \\
\hline Statin & $73(183)$ & $62(55)$ & 0.0583 \\
\hline Aspirin & $77(192)$ & $72(64)$ & 0.3898 \\
\hline Dual Antiplatelet Therapy & $29(72)$ & $27(24)$ & 0.7854 \\
\hline Any anticoagulant & $29(72)$ & $18(16)$ & 0.0493 \\
\hline
\end{tabular}

Values are mean (standard deviation) or \% (n)

There was a significantly higher burden of disease in the beta blocker cohort. Statistically significant differences were noted between the cohorts in terms of preprocedural coronary artery disease/prior CABG, hypertension, dyslipidemia, atrial fibrillation, and diabetes mellitus. There was also a significant increase in preprocedural EuroSCORE, indicating a higher surgical risk in the beta blocker cohort. Baseline and 1-year post-TAVR echocardiographic data for all cohorts is given in Table 2.

There were no significant differences in any variable of interest at both baselines, but there was an increased incidence of severe mitral regurgitation in the control group. The TAVR procedural characteristics are present in Table 3.

No differences were observed in regard to the TAVR approach, length of stay, valve size, or specific type of valve deployed. Finally, the primary and secondary outcomes of this study are presented in Table 4.

Beta blocker usage was associated with an improvement in overall survival after TAVR at 1 year, but not prior to that. Beta blocker use was also associated with a reduction in MACCE in the short term (30 days-6 month) timeframe.
TABLE 2

Echocardiographic data

\begin{tabular}{cccc} 
Variables & Beta-Blocker & Control & P-value \\
\hline Preprocedural & & & \\
Aortic valve area $(\mathrm{VTI})\left(\mathrm{cm}^{2}\right)$ & $0.88(0.35)$ & $0.84(0.36)$ & 0.3991 \\
Peak aortic velocity $(\mathrm{cm} / \mathrm{s})$ & $413.4(66.3)$ & $423.3(61.2)$ & 0.2228 \\
Peak aortic gradient $(\mathrm{mmHg})$ & $70.3(21.0)$ & $73.8(18.5)$ & 0.1682 \\
Mean aortic gradient $(\mathrm{mmHg})$ & $44.4(13.0)$ & $46.6(11.9)$ & 0.1621 \\
Ejection fraction $(\%)$ & $56.6(13.0)$ & $59.7(11.8)$ & 0.0526 \\
Stroke volume $(\mathrm{mL})$ & $85.8(19.1)$ & $85.0(23.0)$ & 0.7756 \\
Moderate aortic regurgitation (\%) & $19(47)$ & $21(19)$ & 0.6407 \\
Severe aortic regurgitation $(\%)$ & $2(6)$ & $6(5)$ & 0.1652 \\
Moderate mitral regurgitation (\%) & $24(59)$ & $19(17)$ & 0.4598 \\
Severe mitral regurgitation $(\%)$ & $3(8)$ & $4(4)$ & 0.5215 \\
1 year post-TAVR & & & \\
Aortic valve area $\left.(\mathrm{VTI})(\mathrm{cm})^{2}\right)$ & $2.00(0.59)$ & $1.95(0.67)$ & 0.6848 \\
Peak aortic velocity $(\mathrm{cm} / \mathrm{s})$ & $220.8(51.3)$ & $213.4(39.5)$ & 0.4896 \\
Peak aortic gradient $(\mathrm{mmHg})$ & $20.5(10.1)$ & $20.1(10.0)$ & 0.8358 \\
Mean aortic gradient $(\mathrm{mmHg})$ & $11.4(5.7)$ & $12.6(6.6)$ & 0.3894 \\
Ejection fraction $(\%)$ & $57.7(13.1)$ & $59.6(12.9)$ & 0.4984 \\
Stroke volume $(\mathrm{mL})$ & $94.9(29.8)$ & $84.5(20.1)$ & 0.1073 \\
Moderate aortic regurgitation (\%) & $14(16)$ & $11(3)$ & 1.000 \\
Moderate mitral regurgitation $(\%)$ & $12(14)$ & $15(4)$ & 0.7473 \\
Severe mitral regurgitation $(\%)$ & $4(4)$ & $15(4)$ & 0.0407 \\
\hline
\end{tabular}

Values are mean (standard deviation) or \%

TABLE 3

Procedural characteristics

\begin{tabular}{|c|c|c|c|}
\hline Variables & $\begin{array}{l}\text { Beta-antagonist } \\
(n=250)\end{array}$ & $\begin{array}{l}\text { Control } \\
(n=89)\end{array}$ & P-value \\
\hline \multicolumn{4}{|c|}{ Approach } \\
\hline Transfermoral & $82(206)$ & $79(70)$ & 0.4315 \\
\hline Transapical & $14(36)$ & $15(13)$ & 1.000 \\
\hline Transaortic & $2(4)$ & $3(3)$ & 0.3847 \\
\hline Trans-subclavian & $1(3)$ & $3(3)$ & 0.0570 \\
\hline Transcaval & $0(1)$ & $0(0)$ & 1.0000 \\
\hline Mean LOS after TAVR (days) & $4.6(7.6)$ & $5.1(5.6)$ & 0.6055 \\
\hline \multicolumn{4}{|c|}{ Valve type } \\
\hline First generation Sapien & $28(69)$ & $26(23)$ & 0.7831 \\
\hline Sapien $X T$ & $15(38)$ & $12(11)$ & 0.6004 \\
\hline Sapien S & $32(80)$ & $38(34)$ & 0.2983 \\
\hline First Generation CoreValve & $22(56)$ & $19(17)$ & 0.5519 \\
\hline CoreValve Evolute & $3(7)$ & $4(4)$ & 0.4881 \\
\hline Mean valve size (mm) & $26.1(2.7)$ & $25.6(2.5)$ & 0.0867 \\
\hline
\end{tabular}

Values are mean (standard deviation) or $n(\%)$

TABLE 4

Primary and secondary outcomes

\begin{tabular}{cccc} 
Variables & $\begin{array}{c}\text { Beta } \\
\text { Antagonist }\end{array}$ & Control & P-value \\
\hline \% Survival > 1 month & $94(236 / 250)$ & $94(84 / 89)$ & 1.0000 \\
$\%$ Survival > 6 months & $89(182 / 204)$ & $87(58 / 67)$ & 0.5165 \\
\% Survival > 1 year & $83(144 / 175)$ & $67(31 / 46)$ & 0.0398 \\
Periprocedural Major Vascular & $8(20)$ & $10(9)$ & 0.5155 \\
Periprocedural Minor Vascular & $9(23)$ & $8(7)$ & 0.8296 \\
Post-TAVR PPM implantation & $9(22)$ & $6(5)$ & 0.4939 \\
Periprocedural Increase in Serum & $5(13)$ & $7(6)$ & 0.5955 \\
Creatinine >1.5x baseline & & & \\
In Hospital & $8(20)$ & $8(7)$ & 1.000 \\
MACCE & $1(2)$ & $0(0)$ & 1.000 \\
MI & $2(5)$ & $4(4)$ & 0.2493 \\
Stroke/TIA & $20(50)$ & $25(22)$ & 0.3670 \\
HF exacerbation & & & \\
Discharge to 30 days & $15(36)$ & $23(19)$ & 0.1343 \\
MACCE & $1(3)$ & $1(1)$ & 1.000 \\
Myocardial Infraction & $1(2)$ & $1(1)$ & 1.000 \\
Stroke/TIA & & &
\end{tabular}




$\begin{array}{cccc}\text { HF exacerbation } & 17(40) & 13(11) & 0.4887 \\ \begin{array}{c}\text { Rehospitalization for any Reason } \\ \text { 30 days- } 6 \text { months }\end{array} & 15(36) & 21(18) & 0.2358 \\ \text { MACCE } & 23(43) & 39(24) & 0.0208 \\ \text { Myocardial Infraction } & 1(1) & 4(3) & 0.0655 \\ \text { Stroke/TIA } & 3(6) & 0(0) & 0.3414 \\ \text { HF exacerbation } & 13(24) & 21(13) & 0.1491 \\ \text { Rehospitalization for any Reason } & 21(39) & 34(21) & 0.0586 \\ \text { 6 months-1 year } & & & \\ \text { MACCE } & 45(31) & 32(11) & 1.000 \\ \text { Myocardial Infraction } & 3(4) & 0(0) & 1.000 \\ \text { Stroke/TIA } & 1(2) & 3(1) & 0.4706 \\ \text { HF exacerbation } & 21(31) & 12(4) & 0.2388 \\ \text { Rehospitalization for any reason } & 29(42) & 26(9) & 0.8538\end{array}$

Values are \% (n); MACCE: Major Adverse Cardiovascular and Cerebrovascular Events; defined as death from any cause, myocardial infarction, rehospitalization, and stroke

\section{DISCUSSION}

In this study, we present evidence that contradicts previous published data and suggests that beta-adrenergic blockers are associated with improved intermediate overall survival, despite an increase in significant comorbidities and predicted surgical risk prior to this procedure. This study also indicates that beta blocker use is associated with a reduction in major adverse cardiovascular and cerebrovascular events in the short-term (30 days to 6 months post-TAVR) post-procedural period, which was driven mostly by a reduction in in the rehospitalization after TAVR. To our knowledge this study is the first to associated beat adrenergic blocker use with improved outcomes following TAVR. Consequently, this study also asserts the safety of beta blocker use in this clinical setting. Given these results, patients without clinical contraindications to beta adrenergic antagonists, TAVR may be a compelling indication for use of one of these agents to potentially improved post-procedural outcomes.

The mechanism by which beta blockers improve outcomes after TAVR is not specific known but given the delay in the onset of the survival benefit of beta blockers seen in this study it is reasonable to assume that the improvement in survival is secondary to the same beneficial mechanisms that beat blocker had in heart failure with reduced ejection fraction (HFrEF). Most notably the suppression of left ventricular remodeling by blocking the sympathetic activation is likely imperative in the improvement in survival, given that new or accelerated hypertension following TAVR is common and occurs in over half of all TAVR patients $(6,7)$. Furthermore, reduction in post-TAVR atrial and ventricular arrhythmias and the anti-ischemic effects of beta blocker also likely play a role in improving outcomes following TAVR and could conceivably fit with the timeline suggest in this study $(8,9)$. It is also significant that beta blocker use was not associated with any increase in heart failure exacerbations. This seems to suggest that implantation of a TAVR valve, may help to overcome the initial changes in inotropy that may occur following TAVR (10).

Despite these encouraging results, our study did not uncover any significant difference in ejection fraction, which has been documented in heart failure with reduced ejection fraction treated with beta blocker. There are multiple potential explanations for this including that TAVR has independently been associated with improvements in ejection fraction, reduction in preload following TAVR to a level that may not be optimal, and preexistent myocardial hypertrophy and fibrosis (5). Furthermore, changes in mitral valve performance could also affect ejection fraction and cardiac output secondary to changes in left ventricle preload.

The study also gives preliminary data to suggest that beta blocker use in TAVR patient may also decrease the prevalence of moderate to severe mitral regurgitation. Previous studies have not found any similar reductions in the amount of regurgitation but have suggested other benefits such as a reduction in left ventricle workload, increased stroke volume, and improved overall survival $(11,12)$. This study was not specifically designed to quantify the clinical impact of the effect of beta blockers on mitral valve function in this study, but this is a potentially significant area for future research and may help to elucidate the mechanism by which beta blocker can improve outcomes following TAVR.

Finally, our study does have some limitations including its retrospective design, single center experience, and variability in the length of post-TAVR follow-up. There were several statistically significant differences in baseline characteristics. However, all of these characteristics seemingly fell in favor of the control group, and therefore are more likely to falsely reduce the true benefit of beta blockers rather than amplify it. This is most notably in the beta blocker cohort having a higher prevalence of preprocedural atrial fibrillation, which has clearly been associated with worse outcomes after TAVR (13). On the other hand, some of the specific differences in baseline characteristics have not been specifically associated with worse outcomes after TAVR including a history of coronary artery disease and a history of coronary artery bypass graft surgery (14).

\section{CONCLUSION}

In this retrospective cohort study, association between the use of betaadrenergic antagonists and improvement in overall survival in patients after TAVR was found in the intermediate time frame. Beta blocker usage was also associated with a reduction in major adverse cardiovascular and cerebrovascular events in the short-term follow-up period. This study suggests that a history of TAVR may be an appropriate compelling indication for the use of beta blockers in an attempt to improve outcomes following TAVR.

\section{ACKNOWLEDGEMENT}

The investigator would like to thank Thomas Haldis, DO and Cornelius Dyke, MD for their help in getting this project started, and Ronda Bolgrean, $\mathrm{RN}$ for her help with data acquisition.

\section{FUNDING}

This research did not receive any specific grant from funding agencies in the public, commercial, or not-for-profit sectors.

\section{DISCLOSURES}

Dr. Klinkhammer has no relationships with industry to disclose.

\section{REFERENCES}

1. Bang CN, Greve AM, Rossebø AB, et al. Antihypertensive treatment with $\beta$-blockade in patients with asymptomatic aortic stenosis and association with cardiovascular events. J Am Heart Assoc 2017;6:e006709.

2. Rossi A, Temporelli PL, Cicoira M, et al. Beta-blockers can improve survival in medically-treated patients with severe symptomatic aortic stenosis. Int J Cardiol 2015;190:15-7.

3. Alrifai A, Sundaravel S, Grajeda E, et al. CRT-700.08 The impact of beta blockers on transcatheter aortic valve replacement outcomes. JACC: Cardiovascular interventions 2018;11.

4. Lindman BR, Otto CM, Douglas PS, et al. Blood pressure and arterial load after transcatheter aortic valve replacement for aortic stenosis. Circ Cardiovasc Imaging 2017;10:e006308.

5. Sato K, Kumar A, Jones BM, et al. Reversibility of cardiac function predicts outcome after transcatheter aortic valve replacement in patients with severe aortic stenosis. J Am Heart Assoc 2017;6:e005798.

6. Kappetein AP, Head SJ, Genereux P, et al. Updated standardized endpoint definitions for transcatheter aortic valve implantation: The Valve Academic Research Consortium-2 consensus document. J Thorac Cardiovasc Surg 2013;145:6-23.

7. Perlman GY, Loncar S, Pollak A, et al. Post-procedural hypertension following transcatheter aortic valve implantation: Incidence and clinical significance. JACC Cardiovasc Interv 2013;6:472-8.

8. Gheorghiade M, Colucci WS, Swedberg K. Betalblockers in chronic heart failure. Circulation 2003;107:1570-5.

9. Kukin ML, Kalman J, Charney RH, et al. Prospective, randomized comparison of effect of long-term treatment with metoprolol or carvedilol on symptoms, exercise, ejection fraction, and oxidative stress in heart failure. Circulation 1999:2645-51.

10. Fearon W, Elmariah S, McAndrew T, et al. TCT-112 Left ventricular ejection fraction improves less after trans-apical transcatheter aortic valve replacement compared to a trans-femoral approach. JACC 2013;62:B36.

11. Stewart RAH, Raffel OC, Kerr AJ, et al. Pilot study to assess the influence of $\beta$-blockade on mitral regurgitant volume and left ventricular work in degenerative mitral valve disease. Circulation 2008;118:1041-6. 
12. Varadarajan P, Joshi N, Appel D, et al. Effect of beta-blocker therapy on survival in patients with severe mitral regurgitation and normal left ventricular ejection fraction. Am J Cardiol 2008;102:611-5.

13. Mojoli M, Gersh BJ, Barioli A, et al. Impact of atrial fibrillation on outcomes of patients treated by transcatheter aortic valve implantation: A systematic review and meta-analysis. Am Heart J 2017;192:64-75.

14. Paradis J, White JM, Généreux P, et al. Impact of coronary artery disease severity assessed with the SYNTAX Score on outcomes following transcatheter aortic valve replacement. J Am Heart Assoc 2017;6:e005070 